\title{
Range effects and lottery pricing
}

\author{
Pavlo R. Blavatskyy • Wolfgang R. Köhler
}

Received: 7 April 2008 / Revised: 11 February 2009 / Accepted: 12 February 2009 /

Published online: 27 February 2009

(C) Economic Science Association 2009

\begin{abstract}
A standard method to elicit certainty equivalents is the Becker-DeGrootMarschak (BDM) procedure. We compare the standard BDM procedure and a BDM procedure with a restricted range of minimum selling prices that an individual can state. We find that elicited prices are systematically affected by the range of feasible minimum selling prices. Expected utility theory cannot explain these results. Nonexpected utility theories can only explain the results if subjects consider compound lotteries generated by the BDM procedure. We present an alternative explanation where subjects sequentially compare the lottery to monetary amounts in order to determine their minimum selling price. The model offers a formal explanation for range effects and for the underweighting of small and the overweighting of large probabilities.
\end{abstract}

Keywords Certainty equivalent $\cdot$ Experiment $\cdot$ Stochastic . Becker-DeGroot-Marschak (BDM) method · Elicitation procedure $\cdot$ Range effects

\section{JEL Classification C91 · D81}

\footnotetext{
We thank seminar participants in Rotterdam and Zürich and at the ESA World Meeting in Rome for helpful comments. We are grateful to Ganna Pogrebna for her assistance with programming the experiment and to Franziska Heusi for her help in organizing the experimental session. Pavlo Blavatskyy acknowledges financial support from the Fund for Support of Academic Development at the University of Zurich. A previous version of this paper was circulated under the title "Lottery Pricing in the Becker-DeGroot-Marschak Procedure".

P.R. Blavatskyy $(\varangle) \cdot$ W.R. Köhler

Institute for Empirical Research in Economics, University of Zurich, Winterthurerstrasse 30, 8006,

Zurich, Switzerland

e-mail: pavlo.blavatskyy@iew.unizh.ch

W.R. Köhler

e-mail: wolfgang.koehler@iew.unizh.ch
} 
A standard method to elicit the certainty equivalent of a risky lottery is the BeckerDeGroot-Marschak (BDM) procedure proposed by Becker et al. (1964). Under the $\mathrm{BDM}$ procedure, individuals are asked to state their minimum selling price for a risky lottery. The experimenter then draws a random number between the lowest and the highest outcome of the lottery. If the price that the individual states is lower than or equal to the drawn number, she receives the drawn number as her payoff. Otherwise she has to play the risky lottery. If preferences satisfy the independence axiom, decisions are not affected by errors, and the reduction-of-compound-lotteries axiom holds, then the BDM procedure elicits the correct certainty equivalent of the lottery.

It is well-known that the BDM procedure does not necessarily provide the correct incentives to reveal the certainty equivalent if preferences violate the independence axiom and individuals take the compound lotteries into account, which they face in a BDM-task (e.g., Karni and Safra 1987). However, if subjects do not consider compound lotteries, the BDM procedure elicits the true certainty equivalents even if the underlying preferences can not be represented by an expected utility functional. Starmer and Sugden (1991) were the first to provide convincing experimental evidence that in binary choice tasks subjects evaluate risky lotteries in isolation and that they ignore the compound lotteries that are generated by the random lottery incentive scheme.

In the BDM-procedure, subjects can usually state any price (or at least any price between the lowest and the highest outcome of the lottery). However, in many pricing decisions exist some upper or lower limits on the possible prices. These limits can be explicit (e.g., the reservation price or the current bid in an auction) or implicit (e.g. the price of the item under consideration at a different firm or the budget constraint).

There exists a large literature on range effects that documents how limits affect prices. To study the effects of limits on prices, we analyze pricing decisions in the standard BDM-task and in a modification of the standard BDM-task - the restricted BDM-task. In a restricted BDM-task, subjects can only state selling prices which lie in an interval that is symmetric around the expected value of the lottery and which includes either the highest or the lowest outcome of the lottery (whichever is closer to the expected value). Similar to the standard BDM-procedure, the random number that is used to determine payoffs in the restricted BDM-task is drawn from the interval of feasible selling prices. Note that a price outside this interval would yield the same distribution of payoffs as a price that is equal to the closest bound of the interval.

If subjects use the same procedure to price lotteries in the restricted and the standard BDM-task, then the elicited prices should be consistent in the following sense. If preferences are deterministic, a subject who states a minimum selling price in the standard BDM-task, which is inside the feasible interval of selling prices in the restricted BDM-task, should state the same price in the restricted BDM-task. Otherwise, the price that she states in the restricted BDM-task should be equal to the closest bound of the interval of feasible prices. If subjects have stochastic preferences, then the percentage of prices that are outside or equal to the bounds in the standard BDM-task should not be statistically different from the percentage of prices that are equal to the bound in the restricted BDM-task.

We run an experiment to compare elicited prices in standard and restricted BDMtasks. Our results can be summarized as follows. The repeated elicitation of minimum 
selling prices via the standard BDM procedure shows that only $16.7 \%$ of subjects consistently state the same minimum selling price for identical lotteries. This indicates that elicited prices are quite stochastic.

A comparison of prices that are elicited in standard and restricted BDM-tasks shows that subjects do not evaluate risky lotteries in isolation. Instead, elicited prices are strongly affected by the interval from which the subject has to choose a price and from which the random number is drawn. This effect depends on the characteristics of the lottery. In a standard BDM-task, subjects state systematically higher (lower) minimum selling prices than in a restricted BDM-task if a two-outcome lottery delivers the highest outcome with probability lower (higher) than 0.5 .

The standard and the restricted BDM-task differ with respect to:

- the interval from which the random number is drawn that determines payoffs

- the range of feasible selling prices that subjects can state.

Hence we analyze two possible explanations:

1. Since the interval from which the random number is drawn differs, subjects face different compound lotteries even if they state the same price in the standard and the restricted BDM-task. If subjects take compound lotteries into account, selling prices can differ across tasks.

2. Since the range of feasible prices differs, range effects can possibly explain the results.

We propose a model of Stochastic Pricing that offers an intuitive explanation for range effects and that explains the systematic differences between prices in standard and restricted BDM-tasks. We consider subjects whose preferences are described by a random utility model. Subjects determine the minimum selling price of a lottery via a sequence of hypothetical binary comparisons between the lottery and different monetary amounts. Depending on whether the amount or the lottery is preferred, the subject decreases or increases the amount to which the lottery is compared. The sequence of comparisons stops if the preferred alternative switches. The selling price that subjects state is the average of the last two amounts to which the lottery has been compared. This model predicts range effects because subjects compare the lottery only to outcomes that are indeed feasible selling prices.

Our model provides an intuitive explanation for the difference in prices across standard and restricted BDM-tasks and for the typical fourfold pattern of riskattitudes (e.g., Tversky and Kahneman 1992). In a companion paper, Blavatskyy and Köhler (2007) test the procedural assumptions of the proposed model of stochastic pricing. Blavatskyy and Köhler (2007) analyze how subjects adjust their stated minimum selling prices under time pressure. They show that the observed price adjustment patterns look exactly like the patterns that are predicted by our stochastic pricing model.

The idea of hypothetical comparisons between the lottery and monetary amounts is similar to the computational model of Johnson and Busemeyer (2005). In their model, pricing a lottery involves a candidate search module (that determines which amount is compared to the lottery) and a comparison module (that specifies how the lottery is compared to the amount). Subjects compare a lottery with an amount via 
evaluating a sequence of hypothetical plays of the lottery. This generates a discrete Markov chain. Subjects declare indifference or preference for one of the alternatives if the Markov process crosses the respective thresholds.

The remainder of this paper is organized as follows. Section 1 describes design, implementation and results of the experiment. Section 2 tests the predictions of different decision theories. Section 3 concludes.

\section{Experimental design and results}

\subsection{Lotteries}

We used 15 risky lotteries that are shown in Table 1. All lotteries have only two outcomes and the lowest outcome is zero. Lotteries 1-3 are the same as in Harbaugh et al. (2003), except that payoffs are in Swiss Francs (CHF) and multiplied by 3.5. Lotteries 4-15 are the same as in lottery set I from Tversky et al. (1990), except that payoffs are in CHF and multiplied by 10 . One CHF was approximately $\$ 0.83$ or $€ 0.61$ at the time of the experiment.

Risky lotteries were described and subsequently played out in terms of the number of red and black cards in a box that contains 100 cards. If the subject drew a black card, she would receive zero. If she drew a red card, she would receive the highest outcome of the lottery. We used a bar to represent the proportion of red and black cards graphically on the computer screen. We used color coding to distinguish different tasks.

\subsection{Elicitation of minimum selling prices}

Subjects were asked twice to state a price for each of the 15 lotteries in Table 1. One price was elicited in a standard BDM-task. The other price was elicited in a restricted BDM-task.

In a standard BDM-task, subjects were endowed with a lottery and they were asked to enter a minimum selling price for the lottery. A screenshot of the standard BDMtask for lottery L1 is shown on the left hand side of Fig. 1. If a standard BDM-task was selected to determine the payoff, a random number was drawn from the interval between zero and the highest outcome of the lottery. If the subject stated a price higher than the randomly drawn number, she would play the lottery. Otherwise she would sell the lottery and receive an amount equal to the randomly drawn number.

Table 1 Lotteries used in the experiment (outcomes are in CHF)

\begin{tabular}{llllllllllllllll}
\hline Lottery & L1 & L2 & L3 & L4 & L5 & L6 & L7 & L8 & L9 & L10 & L11 & L12 & L13 & L14 & L15 \\
\hline Outcome $x_{1}$ & 70 & 70 & 70 & 40 & 160 & 20 & 90 & 30 & 65 & 40 & 400 & 25 & 85 & 20 & 50 \\
Probability $p_{1}$ & 0.1 & 0.4 & 0.8 & 0.97 & 0.31 & 0.81 & 0.19 & 0.94 & 0.5 & 0.89 & 0.11 & 0.94 & 0.39 & 0.92 & 0.5 \\
Outcome $x_{2}$ & 0 & 0 & 0 & 0 & 0 & 0 & 0 & 0 & 0 & 0 & 0 & 0 & 0 & 0 & 0 \\
Probability $p_{2}$ & 0.9 & 0.6 & 0.2 & 0.03 & 0.69 & 0.19 & 0.81 & 0.06 & 0.5 & 0.11 & 0.89 & 0.06 & 0.61 & 0.08 & 0.5 \\
\hline
\end{tabular}



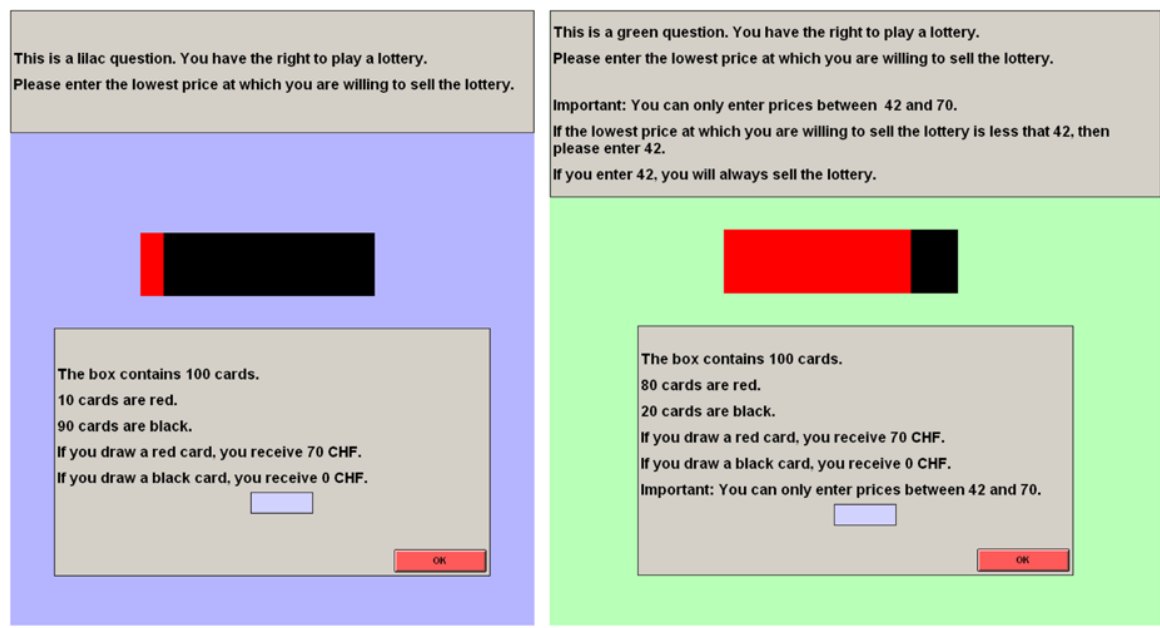

Fig. 1 Screenshots of standard and restricted BDM-tasks (translated from German)

In a restricted BDM-task, subjects were endowed with a lottery and they were asked to enter a selling price. Subjects could only enter prices from a specified interval. For all lotteries we used the interval $\left[\max \left\{0,2 p_{1} x_{1}-x_{1}\right\}, \min \left\{2 p_{1} x_{1}, x_{1}\right\}\right]$, which is symmetric around the expected value of the lottery and includes either the lowest or the highest outcome. A screenshot of the restricted BDM-task for lottery L3 is shown on the right hand side of Fig. 1. If one of the restricted BDM-tasks was selected to determine the earnings, a random number was drawn from the specified interval. If the subject stated a price higher than the drawn number, she would play the lottery. Otherwise she would sell the lottery and receive an amount equal to the drawn number.

\subsection{Implementation of the experiment}

The experiment was conducted in the experimental laboratory of the Institute for Empirical Research in Economics at the University of Zürich. Sixty undergraduates ( 35 male and 25 female) from a variety of majors participated in the experiment. The average age was 22 . There were two sessions with 30 subjects in each session. At the beginning of the experiment, subjects received a copy of the instructions. Instructions included screenshots for the different tasks. Additionally, the experimenter read aloud the instructions. The experiment lasted about 45 minutes (plus 30 minutes to explain the instructions). The experiment was programmed with z-Tree (Fischbacher 2007).

Each subject faced lotteries L1-L15 in a standard and a restricted BDM-task. These decision problems were presented to subjects in random order intermixed with 42 other decision problems that will be analyzed elsewhere. There was no time restriction for decision problems and each subject could continue the experiment at her own pace.

We used a random lottery incentive scheme and physical randomization devices. At the end of the experiment each subject drew a card from a box with cards numbered from 1 to 72 (total number of decision problems). The number on the card 
determined the decision problem which was used to compute the payoff of the subject. If the subject had to play a risky lottery, she had to draw a second card from a box with a specified distribution of red and black cards (we used standard playing cards). Subjects drew the second card outside the main laboratory to preserve the anonymity of payments.

Subjects received a $10 \mathrm{CHF}$ show-up fee and whatever they earned in the experiment. Average earnings were 43.9 CHF (approx. \$36 or €27). The lowest earning was $10 \mathrm{CHF}$, the highest was $133.8 \mathrm{CHF}$. At the end of the experiment, the subjects were asked to complete a short socio-demographic questionnaire.

\subsection{Results}

We begin with the overall consistency of subjects' responses. The restricted and standard BDM-tasks are equivalent for lotteries that involve 50\%-50\% chances. Therefore, we use lotteries L9 and L15 to check the individual consistency of responses. Subjects with deterministic preferences (that are not affected by noise) should state identical minimum selling prices for lotteries L9 and L15 in the standard and restricted BDM-task. Only 10 subjects (16.7\%) showed such consistency for both lotteries. In $40.8 \%$ of cases subjects stated identical minimum selling prices for one of the lotteries (L9 or L15) in standard and restricted BDM-tasks.

We are not aware of any studies that report consistency rates for minimum selling prices. However, we find that our consistency rate for minimum selling prices is significantly lower than consistency rates reported in the literature for binary choice tasks. For example, Hey and Orme (1994) and Ballinger and Wilcox (1997) report that only $25 \%$ and $20.8 \%$ of decisions in binary choice tasks are reversed if subjects face the same decision problem for a second time.

We now investigate if the restrictions on feasible prices in a BDM-task have any effect on the elicited prices. We exclude lotteries L9 and L15 from the current analysis because standard and restricted BDM-tasks are identical for these two lotteries.

Most experimental studies assume that subjects ignore compound lotteries in pricing tasks. Hence as benchmark, consider subjects who ignore compound lotteries. In this case, the fraction of subjects whose minimum selling price for a lottery is outside the interval of feasible prices in the restricted BDM-task is the same as in the corresponding standard BDM-task. Hence the fraction who state a price equal to the bound in the restricted BDM-task is the same as the fraction who state a price outside the interval of feasible prices or equal to the bound in the standard BDM-task.

Table 2 summarizes the results of the experiment. For all lotteries, except L12, the number of subjects, who state a price in the standard BDM-task that lies outside or

Table 2 Number of subjects who state a price outside or equal to the bound of the interval of feasible prices used in the restricted BDM

\begin{tabular}{lrrrrrrrrrrrrrr}
\hline Lottery & L1 & L2 & L3 & L4 & L5 & L6 & L7 & L8 & L10 & L11 & L12 & L13 & L14 & Sum \\
\hline Standard BDM & 35 & 1 & 8 & 21 & 10 & 8 & 16 & 22 & 21 & 22 & 17 & 2 & 14 & 197 \\
Restricted BDM & 12 & 1 & 8 & 9 & 2 & 4 & 2 & 10 & 7 & 7 & 18 & 0 & 4 & 84 \\
\hline
\end{tabular}




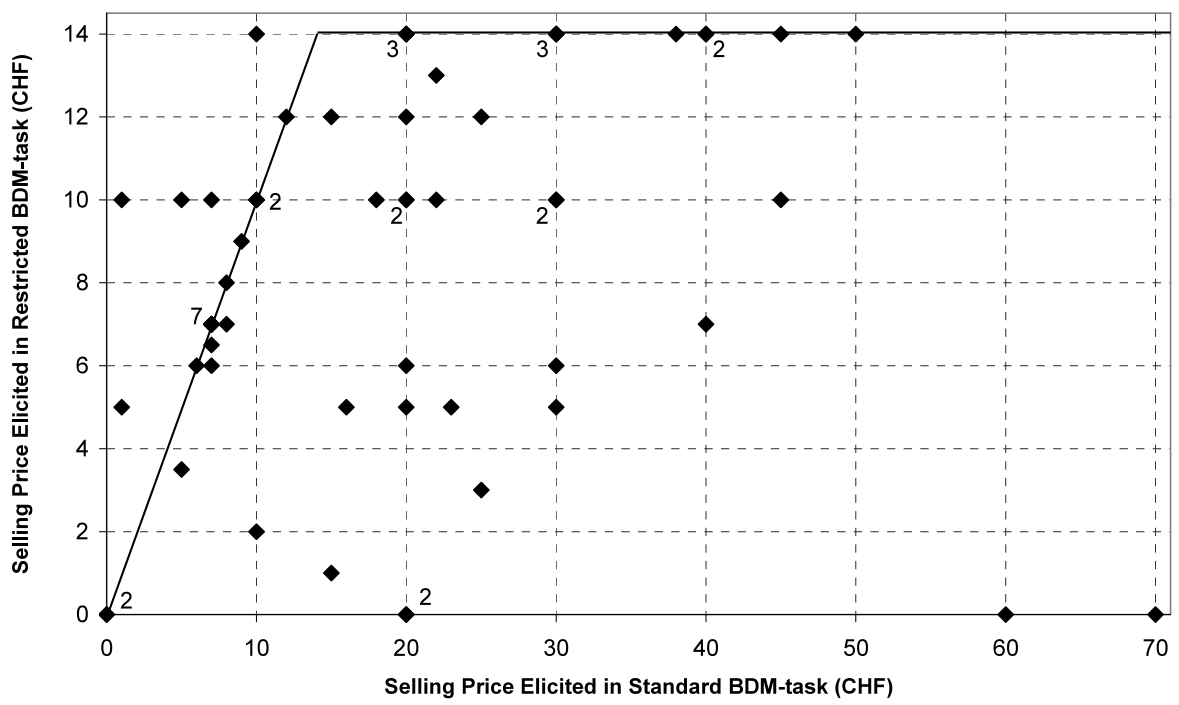

Fig. 2 Selling prices stated by each subject for lottery $\mathrm{L} 1(70,0.1 ; 0,0.9)$

on the bounds of the feasible interval ( $2^{\text {nd }}$ row in Table 2$)$, is higher than the number of subjects, who state a price in the restricted BDM-task that is equal to the bound of the feasible interval (3rd row in Table 2). If subjects used the same method to price lotteries in both BDM-tasks, then the numbers in the second and third row of Table 2 would be the same. Hence, Table 2 suggests that prices elicited through a restricted BDM-task are qualitatively different from those elicited in a standard BDM-task.

Figure 2 shows for each subject the prices in the standard and the restricted BDMtask for lottery L1. In the restricted BDM-task, subjects can state only prices in the interval $[0,14]$. Suppose that subjects ignore compound lotteries. If subjects have deterministic preferences, then all elicited price combinations would lie on the solid line. In other words, if the subject states a price in the standard BDM-task that lies in the interval $[0,14]$, then she states the same price in the restricted BDM-task. If the price in the standard BDM-task is not in the interval $[0,14]$, then the price in the restricted BDM-task is equal to 14 . For 26 out of 60 subjects (43\%), prices for lottery L1 are consistent (i.e., price combinations are located on the solid line in Fig. 2).

If subjects are heterogeneous and have stochastic preferences, then an equal percentage of price combinations should lie to the left and to the right of the solid line. 29 subjects stated a price in the restricted BDM-task that was smaller than the upper bound of the interval and stated a higher price in the standard BDM-task. But only 5 subjects stated a higher price in the restricted BDM-task than in the standard BDMtask. We can strongly reject the null hypothesis of equal proportions $\left(\chi^{2}=9.676\right.$ and $p=0.0018$ ). This striking asymmetry is also observed for the other lotteries where the probability of the highest outcome is less than 0.5 .

The opposite asymmetry is observed for lotteries where the probability of the highest outcome is higher than 0.5. For example, Fig. 3 shows the prices that subjects state in standard and restricted BDM-tasks for lottery L10. In the restricted BDMtask, subjects can state only prices in the interval $[31.2,40]$. For 23 out of 60 subjects 


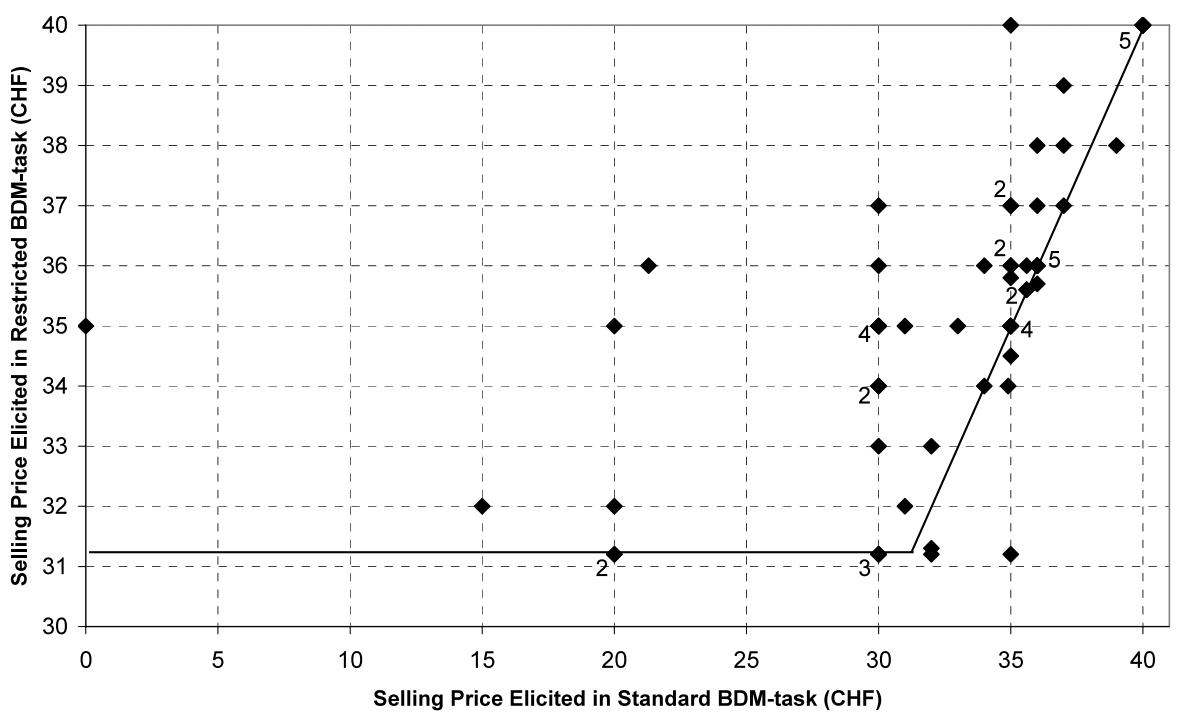

Fig. 3 Selling prices stated by each subject for lottery L10(40, 0.89; 0, 0.11)

(38.3\%), prices are consistent (i.e., price combinations are located on the solid line in Fig. 3). 30 subjects state a price in the restricted BDM-task that is higher than the lower bound and higher than the price elicited in the standard BDM-task. But only 7 subjects state a higher price in the standard BDM-task. Again, we can strongly reject the null hypo-thesis of equal proportions $\left(\chi^{2}=7.913\right.$ and $\left.p=0.0049\right)$. We observe a similar asymmetry for the other lotteries where the probability of the highest outcome is higher than 0.5 .

Our results show that subjects tend to state a higher (lower) price in the restricted BDM-task than in the standard BDM-task if the probability of the highest outcome is higher (lower) than 0.5 . Thus, there is a systematic discrepancy between the prices that are elicited in restricted and standard BDM-tasks.

\section{Theoretical predictions}

\subsection{Aversion to state bounds and the certainty effect}

Before we analyze the predictions of different decision theories, we briefly discuss two possible explanations for the experimental results:

(1) subjects are averse to state bounds

(2) the certainty effect.

(1) Aversion to state bounds refers to the observation that subjects are reluctant to take actions which are extreme relative to the set of feasible actions and instead take some action which is slightly less extreme. In the context of BDM-tasks, this implies that subjects might be reluctant to state one of the bounds of the interval of 
Table 3 Number of subjects who state a price outside the interval of feasible prices or within $10 \%$ of the relevant bound used in the restricted BDM

\begin{tabular}{lcccccccccccccc}
\hline Lottery & L1 & L2 & L3 & L4 & L5 & L6 & L7 & L8 & L10 & L11 & L12 & L13 & L14 & Sum \\
\hline Standard BDM & 35 & 3 & 8 & 22 & 12 & 10 & 16 & 22 & 24 & 24 & 17 & 10 & 21 & 224 \\
Restricted BDM & 13 & 2 & 8 & 11 & 4 & 7 & 8 & 13 & 11 & 13 & 18 & 7 & 13 & 128 \\
\hline
\end{tabular}

feasible prices (even if the bound is their preferred price). Therefore, they state a price close to (but not equal to) the bound. In the standard BDM-task, the bounds of the interval of feasible prices are equal to the lowest and highest outcome of the lottery. In the restricted BDM-task, the interval of feasible prices is symmetric around the expected value and one of the bounds is equal to the lowest or highest outcome of the lottery (whichever is closer to the expected value). To analyze whether aversion to state bounds can explain the experimental results, we consider the bound that differs between the standard and restricted BDM-tasks.

To formalize the idea that subjects are reluctant to state a price equal to the bound and state instead a price close to the bound, we consider prices that differ from the bound by at most $10 \%$ of the length of the interval of feasible prices in the restricted BDM-task. Row 2 in Table 3 contains the number of prices elicited in standard BDMtasks that lie outside the interval or differ from the bound by at most $10 \%$ of the length of the interval. Row 3 in Table 3 contains the number of prices elicited in restricted BDM-tasks that differ from the bound by at most $10 \%$ of the length of the interval.

Table 3 shows the same discrepancy between the elicited prices as Table 2 does, although the differences between row 2 and 3 are smaller (as one would expect since in the standard BDM-task fewer prices lie in the interval than in the restricted BDMtask). Hence, if aversion to state bounds plays a role at all, it can only explain a small part of the discrepancy between the elicited prices in standard and restricted BDM-tasks.

(2) The certainty effect (Kahneman and Tversky 1979) refers to the observation that people seem to overweight outcomes that are certain, relative to outcomes that are merely probable. In the context of our experiment, two prices result in certain outcomes. If the subject states a price equal to the lower bound of the interval of feasible prices, she sells the lottery for sure. If she states a price equal to the upper bound, she plays the lottery for sure. In the restricted BDM-task, one of the bounds differs from the bounds in the standard BDM-task. According to the certainty effect, the percentage of prices in the restricted BDM-task that are equal to this bound should be larger than the percentage of prices that are equal to the bound or outside the feasible interval in the standard BDM-task. Inspection of Table 2 shows immediately that this is not the case.

Of course, this does not imply that aversion to state bounds or the certainty effect do not affect the prices that subjects state in this experiment. However, aversion to state bounds and the certainty effect can neither alone nor in combination explain the discrepancy between the elicited prices in standard and restricted BDM-tasks.

In the remainder of this section, we analyze expected utility theory (EUT) as benchmark and show that EUT cannot explain the data. Then we discuss two possible explanations of the results: compound lotteries and range effects. 


\subsection{Expected utility theory}

According to EUT, the utility of a lottery $L\left(x_{1}, p_{1} ; 0,1-p_{1}\right)$ is $p_{1} u\left(x_{1}\right)$, where $u: \mathbf{R} \rightarrow \mathbf{R}$ is a non-decreasing Bernoulli utility function that is normalized so that $u(0)=0$. The certainty equivalent $C E_{L}$ of $L$ is implicitly defined by $u\left(C E_{L}\right)=$ $p_{1} u\left(x_{1}\right)$.

Consider a standard BDM-task. A subject who states a minimum selling price $x \in\left[0, x_{1}\right]$ for lottery $L$ faces a compound lottery that yields the simple lottery $L$ with probability $x / x_{1}$ (i.e., when the number that is drawn at random from the interval $\left[0, x_{1}\right]$ is smaller or equal to $x$ ). Additionally, the compound lottery yields every outcome in the interval $\left[x, x_{1}\right]$ with equal probability. A subject, who states a minimum selling price $x$, obtains utility $U_{S}(x)=\left(x / x_{1}\right) p_{1} u\left(x_{1}\right)+\left(1 / x_{1}\right) \int_{x}^{x_{1}} u(y) d y$. The price $x_{S}$ that maximizes $U_{S}$ is the solution to $d U_{S}(x) / d x=0$. Hence $u\left(x_{S}\right)=$ $p_{1} u\left(x_{1}\right)$. Thus, $x_{S}=C E_{L}$ and expected utility maximizers reveal their certainty equivalent in a standard BDM-task.

In a restricted BDM-task, subjects can only state prices in the interval $[\underline{x}, \bar{x}]$, where $\underline{x}=x_{1} \max \left\{0,2 p_{1}-1\right\}$ and $\bar{x}=x_{1} \min \left\{2 p_{1}, 1\right\}$ are the bounds of the interval of feasible prices. A subject, who states price $x$ for lottery $L$, faces a compound lottery that yields the simple lottery $L$ with probability $(x-\underline{x}) /(\bar{x}-\underline{x})$. Additionally, the compound lottery yields every outcome in the interval $[x, \bar{x}]$ with equal probability. If a subject states price $x$, the utility of the compound lottery is

$$
U_{R}(x)=\frac{(x-\underline{x}) \cdot p_{1} u\left(x_{1}\right)+\int_{x}^{\bar{x}} u(y) d y}{\bar{x}-\underline{x}} .
$$

If there exists $x_{R} \in[\underline{x}, \bar{x}]$ such that $d U_{R}\left(x_{R}\right) / d x=0$, then $x_{R}$ is the price that maximizes $U_{R}$. Hence $u\left(x_{R}\right)=p_{1} u\left(x_{1}\right)$ and, therefore, $x_{R}=C E_{L}$. If there exists no $x_{R} \in[\underline{x}, \bar{x}]$ such that $d U_{R}\left(x_{R}\right) / d x=0$, then there are two cases. If $p_{1}<1 / 2$, then $\underline{x}$ is equal to the lowest outcome of the lottery and $d U_{R}(x) / d x>0$ for every $x \in[\underline{x}, \bar{x}]$. Hence the price $x_{R}$ that maximizes $U_{R}$ is equal to $\bar{x}$. If $p_{1}>1 / 2$, then $\bar{x}$ is equal to the highest outcome of the lottery and $d U_{R}(x) / d x<0$ for every $x \in[\underline{x}, \bar{x}]$. Hence the price $x_{R}$ that maximizes $U_{R}$ is equal to $\underline{x}$.

Thus, in a restricted BDM-task expected utility maximizers state a price that is equal to their certainty equivalent if the certainty equivalent lies in interval $[\underline{x}, \bar{x}]$. Otherwise, they state the price $2 x_{1} p_{1}$ if $p_{1}<1 / 2$ and $\left(2 p_{1}-1\right) x_{1}$ if $p_{1}>1 / 2$.

We are interested to test whether a stochastic version of EUT can explain the discrepancy of elicited prices in the restricted and the standard BDM-tasks. We consider heterogeneous subjects who have stochastic preferences where each preference relation can be described by EUT. To test the predictions of EUT, we compute a sample of prices that are predicted by EUT for the restricted BDM-task. To compute the sample of predicted prices, we take the prices that are elicited in the standard BDM-task and replace prices that lie outside the interval of feasible prices with the respective bound and leave the other prices unchanged.

We compare the elicited and predicted prices for each subject and each lottery. Predicted prices are generated by applying the prediction of EUT. It follows immediately that according to EUT, for each subject and for each lottery, the probability 
Table 4 Results of a sign test of the prediction of expected utility theory

\begin{tabular}{lll}
\hline & Lotteries with $p_{1}<1 / 2$ & Lotteries with $p_{1}>1 / 2$ \\
\hline Actual price is above predicted & $25.8 \%$ & $39.8 \%$ \\
Actual price is below predicted & $46.7 \%$ & $19.3 \%$ \\
$p$-value for sign test & 0.000 & 0.000 \\
\hline
\end{tabular}

that the predicted price is smaller than the elicited price in the restricted BDM-task is equal to the probability that the predicted price is larger than the elicited price. We use a sign-test to analyze how predicted and elicited prices differ. For each subject and lottery, we compute the difference between the elicited price in the restricted BDM-task and the predicted price according to EUT. The null-hypothesis is that the differences are drawn from a distribution with median zero.

Table 4 summarizes the results. The second and third row of Table 4 show the percentage of elicited prices in a restricted BDM-task that are higher and lower than the predicted price. The sign-test shows that we can reject the null-hypothesis that it is equally likely that the price elicited in a restricted BDM-task is smaller respectively larger than the predicted price. For lotteries with $p_{1}<1 / 2\left(p_{1}>1 / 2\right)$, minimum selling prices stated in a restricted BDM-task are systematically below (above) predicted prices.

Table 4 shows that we can reject EUT as explanation for the discrepancy between elicited prices in standard and restricted BDM-tasks. Furthermore, we can also reject any other decision theory that predicts the same consistency of selling prices in standard and restricted BDM-tasks.

\subsection{Rank-dependent utility theory (RDEU) and cumulative prospect theory}

If preferences violate the independence axiom and subjects take compound lotteries into account, then optimal prices in standard and restricted BDM-tasks can differ systematically. In this section, we investigate the predictions of one popular generalization of expected utility theory that does not assume independence-the rankdependent utility model (RDEU) proposed by Quiggin (1981). The predictions of RDEU coincide with the predictions of cumulative prospect theory (Tversky and Kahneman 1992) when all lottery outcomes are above or equal to the reference point.

According to RDEU, the utility of lottery $L\left(x_{1}, p_{1} ; 0,1-p_{1}\right)$ is $w\left(p_{1}\right) \cdot u\left(x_{1}\right)$, where $w:[0,1] \rightarrow[0,1]$ is a non-decreasing probability weighting function that satisfies $w(0)=0$ and $w(1)=1$. The certainty equivalent $C E_{L}$ is implicitly defined by $u\left(C E_{L}\right)=w\left(p_{1}\right) \cdot u\left(x_{1}\right)$.

An individual who states a minimum selling price $x$ in a standard BDM-task obtains utility $U_{S}^{\mathrm{RDEU}}(x)=w\left(p_{1} x / x_{1}\right) \cdot u\left(x_{1}\right)+\int_{x}^{x_{1}} w^{\prime}\left(1-y / x_{1}+p_{1} x / x_{1}\right) \times$ $u(y) d y$. The minimum selling price $x_{S}$ which maximizes $U_{S}^{\mathrm{RDEU}}$ is the solution to $d U_{S}^{\mathrm{RDEU}}(x) / d x=0$. An individual who states a minimum selling price $x$ in a restricted BDM-task obtains utility $U_{R}^{\mathrm{RDEU}}(x)=w\left(\frac{x-\underline{x}}{\bar{x}-\underline{x}} p_{1}\right) u\left(x_{1}\right)+$ $\int_{x}^{\bar{x}} w^{\prime}\left(\frac{\bar{x}-y+p_{1}(x-\underline{x})}{\bar{x}-\underline{x}}\right) u(y) d y$. Let $x_{R} \in[\underline{x}, \bar{x}]$ be the price that maximizes $U_{R}^{\mathrm{RDEU}}$. 


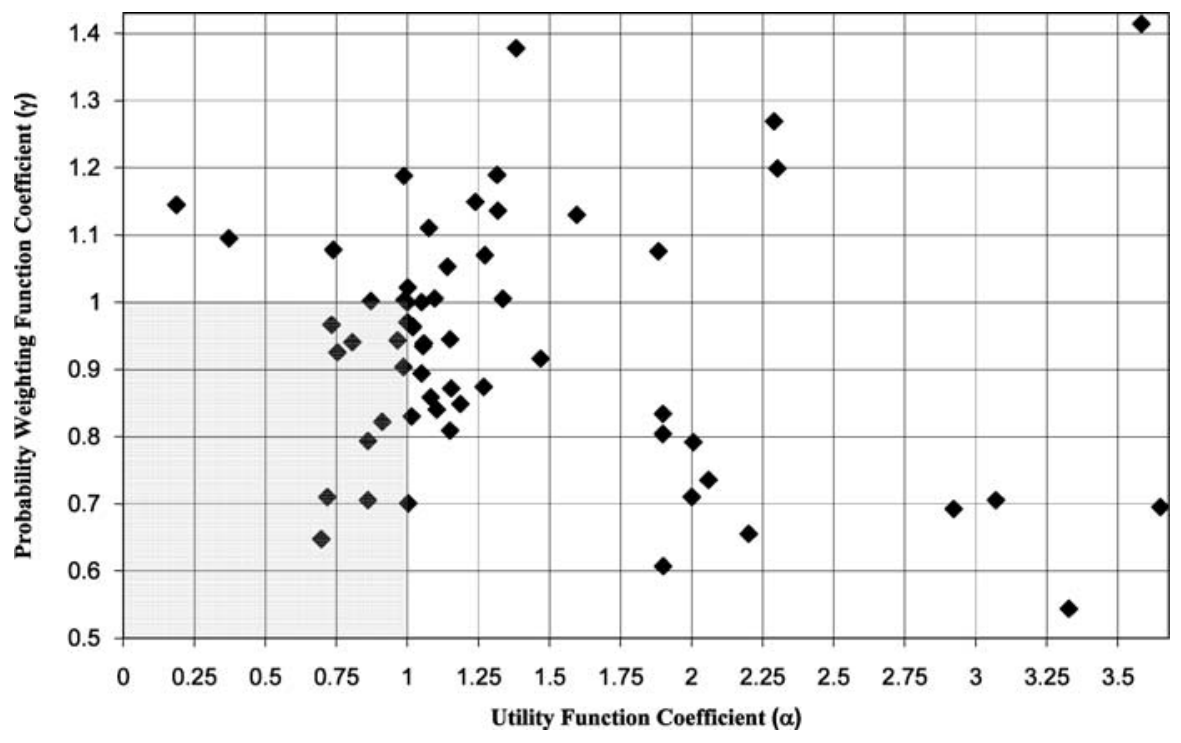

Fig. 4 Scatterplot of the estimated parameters of RDEU $(N=60)$ if subjects take compound lotteries into account

We estimated the parameters of RDEU separately for each subject using utility function $u(x)=x^{\alpha}$ and probability weighting function $w(p)=p^{\gamma} /\left(p^{\gamma}+\right.$ $\left.(1-p)^{\gamma}\right)^{1 / \gamma}$. The coefficients $\alpha$ and $\gamma$ are estimated to minimize the weighted sum of squared errors $S S E=\sum_{i=1}^{15}\left[\left(x_{S}^{i}-D_{S}^{i}\right) / x_{1}^{i}\right]^{2}+\sum_{i=1}^{15}\left[\left(x_{R}^{i}-D_{R}^{i}\right) / x_{1}^{i}\right]^{2}$, where $D_{S}^{i}$ and $D_{R}^{i}$ are the prices that a subject stated for lottery $i$ in a standard and restricted BDM-tasks and $x_{S}^{i}$ and $x_{R}^{i}$ are the corresponding predictions of RDEU. Non-linear unconstrained optimization was implemented in the Matlab 7.2 package (based on the Nelder-Mead simplex algorithm).

Figure 4 shows the scatterplot of the estimated parameters of RDEU for all 60 subjects. Median estimated parameters are $\alpha=1.10$ and $\gamma=0.94 .42$ out of 60 subjects $(70 \%)$ have a typical inverse S-shaped weighting function $(\gamma<1)$ and 22 out of 60 subjects $(37 \%)$ have a typical concave utility function $(\alpha<1)$. For 12 subjects $(20 \%)$ the estimated coefficients are in the range of typical parameterizations of RDEU i.e. $0<\alpha<1$ (concave utility function) and $0<\gamma<1$ (inverse S-shaped weighting function), shown as a shaded area in Fig. 4. For a majority of subjects, the estimated parameters of RDEU lie outside the range of typical parameterizations. This finding is not surprising because we assume that subjects take compound lotteries into account. In contrast, RDEU is typically estimated on the assumption that subjects ignore compound lotteries.

\subsection{A model of stochastic pricing}

In this section, we propose an alternative explanation for the discrepancy of prices in standard and restricted BDM-tasks. We analyze a model of stochastic pricing where individuals compare the lottery to different monetary amounts to determine the minimum selling price. This model offers a behavioral foundation of range effects. 
In Sect. 1.4, we have shown that the consistency rates of elicited selling prices for identical lotteries are quite low. To account for low consistency rates, we consider individuals with stochastic preferences. Each individual is characterized by a set $\Pi$ of rational preference relations on the space of lotteries and a probability measure $\eta$ on $\Pi$. Preferences are described by a pair $(\eta, \Pi)$. If an individual faces a binary choice problem, she draws a preference relation $\succsim_{\rho} \in \Pi$ according to $\eta$ and chooses according to the realized $\succsim_{\rho}$. To determine the minimum selling price of a lottery, the individual compares the lottery to different monetary amounts which are drawn from the set of possible prices $S_{L}$.

In a standard BDM-task, the set of possible prices is the interval between the lowest and the highest outcome. Hence, for lottery $L\left(x_{1}, p_{1} ; 0,1-p_{1}\right)$ we have $S_{L}=$ $\left[0, x_{1}\right]$. Wilcox $(1994$, p. 318) provides evidence that subjects search between the highest and the lowest outcome of a lottery to find their minimum selling price in a standard BDM-task. In a restricted BDM-task, the set of possible prices is equal to the interval of feasible prices.

To find the minimum selling price of lottery $L$, individuals first draw an amount $x \in S_{L}$ at random. In the second step, they draw a preference relation $\succsim_{\rho} \in \Pi$ according to probability measure $\eta$ and compare $x$ to the lottery $L$. If $x \sim{ }_{\rho} L$ then $x$ is stated as the minimum selling price of $L$. If $x \succ_{\rho} L$, then step 2 is repeated with $x$ being replaced by $\max \left\{x-\Delta, \min S_{L}\right\}$, where $\Delta>0$ is the step size by which the amount is adjusted. If $L \succ_{\rho} x$, then step 2 is repeated with $x$ being replaced by $\min \{x+\Delta$, $\max S_{L}$. Note that individuals draw a new preference relation each time when they compare a lottery to a monetary amount. The sequence of binary comparisons ends if the preferred alternative switches. In this case, the minimum selling price is the average of the last two amounts to which the lottery has been compared.

For example, consider an individual who prices the lottery $L(70,0.1 ; 0,0.9)$. The set of possible prices is [0,70]. Suppose that the individual starts the sequence of binary comparisons with $x=20$ and the step size is $\Delta=5$. If $x \succ L$, she then compares the lottery with $x=15$. If she still prefers $x$, in the next step she compares the lottery with $x=10$. If she now prefers the lottery over $x=10$, she states a minimum selling of 12.5 .

The stochastic pricing model describes the determination of the minimum selling price as a grid search where the lottery is compared to different monetary amounts. If individuals use such a simple grid search, then the price that an individual states in a standard and restricted BDM-task can be described as random variable whose distribution depends on the probability measure $\eta$ over preference relations, on the step-size $\Delta$, and the set of possible prices $S_{L}$. Consider the simplest possible case of a two outcome lottery $L\left(x_{1}, p_{1} ; 0,1-p_{1}\right)$ when individuals are "on average" risk neutral, i.e. they are just as likely to choose amount $p_{1} x_{1}-\delta$ over lottery $L$ as they are likely to choose $L$ over amount $p_{1} x_{1}+\delta$. In this case, the median selling price in a restricted BDM-task is just the expected value $p_{1} x_{1}$ of the lottery. However, the median selling price in a standard BDM-task is higher (lower) than the expected value of the lottery for lotteries with $p_{1}<1 / 2\left(p_{1}>1 / 2\right)$, at least if preferences are sufficiently random relative to the step size $\Delta$.

Intuitively, if $p_{1}<1 / 2$, then an individual is more likely to start the grid search in a standard BDM-task with an amount $x$ that is higher than the expected value of 


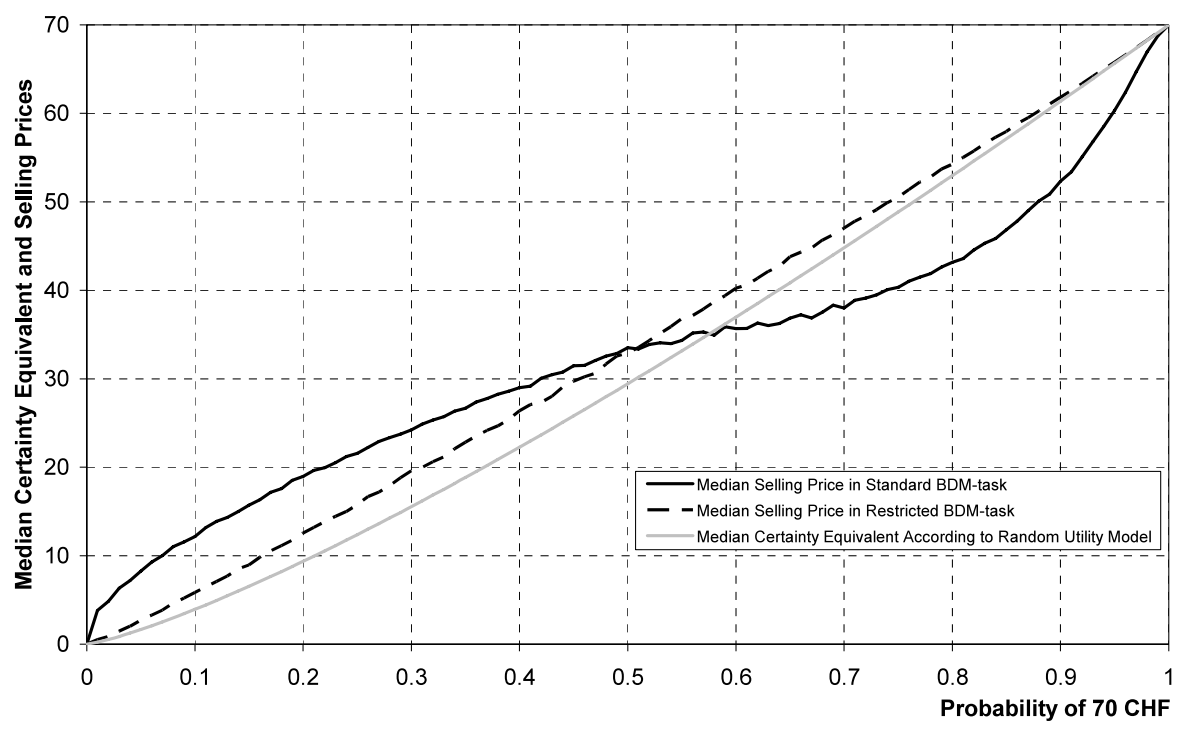

Fig. 5 Monte Carlo simulation of median prices for lottery $L\left(70, p_{1} ; 0,1-p_{1}\right)$ when preferences are represented by the random utility function $u(x)=x^{1-r} /(1-r), r \sim N(0.2,0.4)$ and prices are determined by a grid search with step size $\Delta=1 \mathrm{CHF}$

the lottery. If preferences are sufficiently stochastic relative to the step size $\Delta$, this individual is then also more likely to end the grid search at an amount higher than the expected value of the lottery. The reverse holds for lotteries with $p_{1}>1 / 2$. Thus, a simple model of stochastic pricing explains both the fourfold pattern of risk attitudes and the systematic discrepancies between elicited prices in standard and restricted BDM-tasks that we observed in our experiment.

For example, Fig. 5 shows the median certainty equivalent and a Monte Carlo simulation of the median selling prices for lottery $L\left(70, p_{1} ; 0,1-p_{1}\right)$ when probability $p_{1}$ is varied between 0 and 1 . We assume that every preference relation is represented by a constant relative risk aversion utility function $u(x)=x^{1-r} /(1-r)$. We assume that the coefficient $r$ is normally distributed with mean 0.2 and standard deviation 0.4 . The price for the lottery is determined by a simple grid search with the step size $\Delta=1 \mathrm{CHF}$. For each value of probability $p_{1}$ we conducted $10^{4}$ simulations of prices in standard and restricted BDM-tasks. The median values of the simulated prices are shown in Fig. 5.

Figure 5 shows that median prices in a standard BDM-task are systematically higher (lower) than median prices in a restricted BDM-task for lotteries with $p_{1}<$ $1 / 2\left(p_{1}>1 / 2\right)$. The reason for the different prices is not that subjects face different compound lotteries in the two tasks. Instead, the reason is that the monetary amounts that are compared to the lottery are drawn from different intervals. Additionally, our model of stochastic lottery pricing explains the risk-seeking (risk-averse) decisions in standard BDM-pricing tasks for lotteries with a low (high) probability of a gain although preferences in this example are captured by a random expected utility model.

We estimate the proposed model of stochastic pricing on our experimental data. We assume that the preferences of every subject are represented by a constant relative 
risk aversion utility function $u(x)=x^{1-r} /(1-r)$ with coefficient $r$ being normally distributed with mean $\mu$ and standard deviation $\sigma$. Note that in this case, the probability that a subject chooses lottery $L\left(x_{1}, p_{1} ; 0,1-p_{1}\right)$ over amount $x \in\left[0, x_{1}\right]$ for sure is

$$
\operatorname{Pr}\{L \succ x\}= \begin{cases}1, & x=0 \\ \Phi_{\mu, \sigma}\left(1+\log _{x_{1} / x} p_{1}\right), & x \in\left(0, x_{1}\right) \\ 0, & x=x_{1}\end{cases}
$$

where $\Phi_{\mu, \sigma}$ (.) denotes the cumulative distribution function of a normal distribution with mean $\mu$ and standard deviation $\sigma$. The probability that a subject chooses amount $x$ over lottery $L$ is $\operatorname{Pr}(x \succ L)=1-\operatorname{Pr}(L \succ x)$, where $\operatorname{Pr}(L \succ x)$ is defined in (1).

If a subject starts the sequence of hypothetical binary comparisons from amount $x$, then the likelihood that this subject reveals selling price $z$ is approximated by

$$
\widetilde{\operatorname{Pr}}(x, z)=\left\{\begin{array}{l}
\operatorname{Pr}(L \succ x) \times \cdots \times \operatorname{Pr}(L \succ x+k \Delta) \cdot \operatorname{Pr}\left(\min \left\{x+(k+1) \Delta, x_{1}\right\} \succ L\right), \\
\quad x<z \\
\operatorname{Pr}(x \succ L) \times \cdots \times \operatorname{Pr}(x-m \Delta \succ L) \cdot \operatorname{Pr}(L \succ \max \{x-(m+1) \Delta, 0\}), \\
\quad x \geq z
\end{array}\right.
$$

where $k \in\{0,1, \ldots\}$ is the highest number such that $x+k \Delta<z, m \in\{0,1, \ldots\}$ is the highest number such that $x-m \Delta \geq z$ and $\Delta$ is the step size of the grid search. For every subject, random utility parameters $\mu$ and $\sigma$ are estimated to maximize the total log-likelihood

$$
\begin{aligned}
L L= & \sum_{i=1}^{15} \ln \left(\frac{1}{x_{1}^{i} / \Delta+1} \sum_{j=1}^{x_{1}^{i} / \Delta+1} \tilde{\operatorname{Pr}}\left(\Delta(j-1), D_{S}^{i}\right)\right) \\
& +\sum_{i=1}^{15} \ln \left(\frac{1}{\left(\bar{x}^{i}-\underline{x}^{i}\right) / \Delta+1} \sum_{j=1}^{\left(\bar{x}^{i}-\underline{x}^{i}\right) / \Delta+1} \tilde{\operatorname{Pr}}\left(\Delta(j-1), D_{R}^{i}\right)\right)
\end{aligned}
$$

where the step size $\Delta$ is taken to be $10 \%$ of the interval of feasible prices i.e. $\Delta=$ $x_{1} / 10$ in the standard BDM-task and $\Delta=(\bar{x}-\underline{x}) / 10$ in the restricted BDM-task. Across all 60 subjects, median estimated parameters turned out to be $\mu=-0.86$ and $\sigma=1.46$.

To compare the fit of the stochastic pricing model with that of RDEU, we run a Monte Carlo simulation where we use the estimated parameters of the stochastic pricing model to generate minimum selling prices. For each subject and each decision we run $10^{4}$ simulations with the estimated random utility parameters $\mu$ and $\sigma$ and the step size $\Delta=10 \%$. We find that simulated mean prices are close to the prices predicted by RDEU. For 28 out of 60 subjects (46.7\%), the correlation coefficient between simulated mean prices from the stochastic pricing model and prices predicted by RDEU is higher than 0.95 , which indicates that two models make nearly identical predictions.

The fit of the stochastic pricing model is similar to that of RDEU. If we compare SSE for the stochastic pricing model with SSE for the RDEU prediction, 29 out of 
60 subjects $(48.3 \%)$ have a lower SSE for the stochastic pricing model. However, the difference between the SSE is small. For 18 subjects, the difference of the SSE of the two models is less than 5\%. Among the remaining subjects, 20 (22) subjects have a lower SSE for the stochastic pricing model (for RDEU). Thus, the stochastic pricing model explains the experimental results about as well as RDEU, when RDEU takes compound lotteries into account.

\subsection{Out-of-sample prediction}

Both RDEU and the stochastic pricing model achieve a similar goodness of fit when they are estimated on the complete data set (prices stated in standard and restricted BDM-tasks). However, as economists, we are ultimately interested in how good models predict decisions. Therefore, we also compare the two models by the quality of their out-of sample predictions. For every subject, we estimate the parameters of both models using only the elicited prices from the standard BDM-task. Then we use the estimated parameters to predict the minimum selling price that this subject states in the restricted BDM-task. Note that we can not estimate parameters using only prices from the restricted BDM-task. If subjects state the bound in the restricted BDM-task, then parameters can not be determined if we do not have information about the price that they state in the standard BDM-task.

We estimate RDEU separately for each subject using the elicited prices from the standard BDM-task and utility function $u(x)=x^{\alpha}$ and probability weighting function $w(p)=p^{\gamma} /\left(p^{\gamma}+(1-p)^{\gamma}\right)^{1 / \gamma}$. The coefficients $\alpha$ and $\gamma$ are estimated to minimize the weighted sum of squared errors $S S E=\sum_{i=1}^{15}\left[\left(x_{S}^{i}-D_{S}^{i}\right) / x_{1}^{i}\right]^{2}$, where $D_{S}^{i}$ is the price that a subject stated for lottery $i$ in a standard BDM-task and $x_{S}^{i}$ is the corresponding prediction of RDEU (as described in Sect. 2.3). Given the estimated parameters $\hat{\alpha}$ and $\hat{\gamma}$ we compute the predicted selling price for lottery $i$ in a restricted BDM-task $x_{R}^{i}(\hat{\alpha}, \hat{\gamma})$ as described in Sect. 2.3. Given these predicted prices we compute the sum of squared errors for the RDEU prediction, i.e., $S S E=\sum_{i=1}^{15}\left[\left(x_{R}^{i}(\hat{\alpha}, \hat{\gamma})-D_{R}^{i}\right) / x_{1}^{i}\right]^{2}$.

We estimate the proposed model of stochastic pricing separately for each subject using the elicited prices from the standard BDM-task and utility function $u(x)=$ $x^{1-r} /(1-r)$ where coefficient $r$ is normally distributed with mean $\mu$ and standard deviation $\sigma$. For every subject, random utility parameters $\mu$ and $\sigma$ are estimated to maximize log-likelihood $L L=\sum_{i=1}^{15} \ln \left(\frac{1}{x_{1}^{i} / \Delta+1} \sum_{j=1}^{x_{1}^{i} / \Delta+1} \widetilde{\operatorname{Pr}}\left(\Delta(j-1), D_{S}^{i}\right)\right)$ where the step size $\Delta$ is $10 \%$ of the interval of feasible prices and $\widetilde{\operatorname{Pr}}\left(\Delta(j-1), D_{S}^{i}\right)$ is defined as in Sect. 2.4. For each subject and each lottery we then run $10^{4}$ simulations of prices stated in the restricted BDM-task given the estimated random utility parameters $\hat{\mu}$ and $\hat{\sigma}$ and the step size $\Delta=10 \%$. Taking the mean values $\bar{x}_{R}^{i}(\hat{\mu}, \hat{\sigma})$ of these $10^{4}$ Monte Carlo simulations, we compute the sum of squared errors for the model of stochastic pricing, i.e., $S S E=\sum_{i=1}^{15}\left[\left(\bar{x}_{R}^{i}(\hat{\mu}, \hat{\sigma})-D_{R}^{i}\right) / x_{1}^{i}\right]^{2}$.

A comparison of the sum of squared errors for predicted prices in restricted BDMtasks clearly shows that the stochastic pricing model predicts prices more accurately. For 39 out of 60 subjects $(65 \%)$ the model of stochastic pricing has a lower sum of squared errors than RDEU. For 26 subjects (43\%) the sum of squared errors of the 
stochastic pricing model is at least $20 \%$ smaller than the sum of squared errors of the RDEU prediction. But only for 8 subjects $(13 \%)$ the sum of squared errors of the RDEU prediction is at least $20 \%$ smaller than the sum of squared errors of the prediction of the stochastic pricing model. Therefore, the model of stochastic pricing appears to have a better out-of-sample forecasting power than RDEU.

\section{Conclusion}

We study lottery pricing under the BDM procedure. We compare prices that are elicited in standard BDM-tasks with those elicited in restricted BDM-tasks. The two tasks differ in one important aspect. In a standard BDM-task, subjects can state prices between the highest and the lowest outcome of the lottery. In a restricted BDM-task, subjects can state prices that lie within a smaller interval that is symmetric around the expected value of the lottery. The interval imposes a lower (upper) bound on prices for lotteries when the probability $p$ of a gain is higher (lower) than 0.5 . We observe strong range effects - a highly significant discrepancy between the prices for the same lottery in standard and restricted BDM-tasks. Subjects state systematically higher (lower) prices in the restricted BDM-task for lotteries with $p>0.5(p<0.5)$.

Our results have several implications for future research. It appears that prices elicited in standard BDM-tasks are systematically too high (low) if the chance of a gain is low (high). This supports recent findings of Bateman et al. (2007), who argue that some empirical anomalies such as the preference reversal phenomenon may be an artefact of the BDM-task and that their frequency is significantly reduced if lottery prices are elicited through different techniques.

The proposed model of stochastic pricing provides a behavioral foundation for range effects that are frequently observed in experiments. The discrepancy between prices in standard and restricted BDM-tasks can be interpreted as the result of range effects. The stochastic pricing model provides a simple and intuitive explanation for why we observe a range effect. The range effect occurs because the restriction on the interval of feasible prices affects the set of feasible prices that may be compared to the lottery in a sequence of binary comparisons.

Finally, our experimental results have important methodological implications for experimental economics. Inconsistencies in minimum selling prices elicited in standard and restricted BDM-tasks indicate that a classical BDM procedure is highly sensitive to range effects. Hence, experimental economists should be aware that prices elicited in the BDM procedure are distorted by range effects and they should take this bias into account. By using the restricted BDM-task a researcher can reduce such range affects.

Additionally, the restricted BDM-task provides higher incentives for reporting true certainty equivalents. If a subject misreports his true price by the same amount in standard and restricted BDM-tasks, his expected utility is $0.5 / \min \{p, 1-p\}$ times lower in the restricted BDM-task compared to the standard BDM-task (provided that the true price lies within the feasible interval). However, the restricted BDM-task has an obvious drawback - a researcher cannot observe minimum selling prices outside 
the (preselected) feasible interval. Moreover, the restricted BDM-task may drift subjects to a risk-neutral behavior. Thus, we would not recommend using the restricted BDM-task as a superior method over the standard BDM procedure.

\section{References}

Ballinger, T. P., \& Wilcox, N. T. (1997). Decisions, error and heterogeneity. Economic Journal, 107, 10901105.

Bateman, I., Day, B., Loomes, G., \& Sugden, R. (2007). Can ranking techniques elicit robust values?. Journal of Risk and Uncertainty, 34, 49-66.

Becker, G. M., DeGroot, M. H., \& Marschak, J. (1964). Measuring utility by a single-response sequential method. Behavioral Science, 9, 226-232.

Blavatskyy, P., \& Köhler, W. (2007). Lottery pricing under time pressure. IEW working paper.

Fischbacher, U. (2007). z-tree: Zurich toolbox for ready-made economic experiments. Experimental Economics, 10(2), 171-178.

Harbaugh, W. T., Krause, K., \& Vesterlund, L. (2003). Prospect theory in choice and Pricing tasks. Working paper.

Hey, J., \& Orme, C. (1994). Investigating generalisations of expected utility theory using experimental data. Econometrica, 62, 1291-1326.

Johnson, J. G., \& Busemeyer, J. R. (2005). A dynamic, stochastic, computational model of preference reversal phenomena. Psychological Review, 112, 841-861.

Kahneman, D., \& Tversky, A. (1979). Prospect theory: an analysis of decision under risk. Econometrica, 47, 263-291.

Karni, E., \& Safra, Z. (1987). Preference reversal" and the observability of preferences by experimental methods. Econometrica, 55, 675-685.

Quiggin, J. (1981). Risk perception and risk aversion among Australian farmers. Australian Journal of Agricultural Recourse Economics, 25, 160-169.

Starmer, C., \& Sugden, R. (1991). Does the random-lottery incentive system elicit true preferences? An experimental investigation. American Economic Review, 81, 971-978.

Tversky, A., Slovic, P., \& Kahneman, D. (1990). The causes of preference reversal. American Economic Review, 80, 204-217.

Tversky, A., \& Kahneman, D. (1992). Advances in prospect theory: Cumulative representation of uncertainty. Journal of Risk and Uncertainty, 5, 297-323.

Wilcox, N. (1994). On a lottery pricing anomaly: time tells the tale. Journal of Risk and Uncertainty, 8, 311-324. 